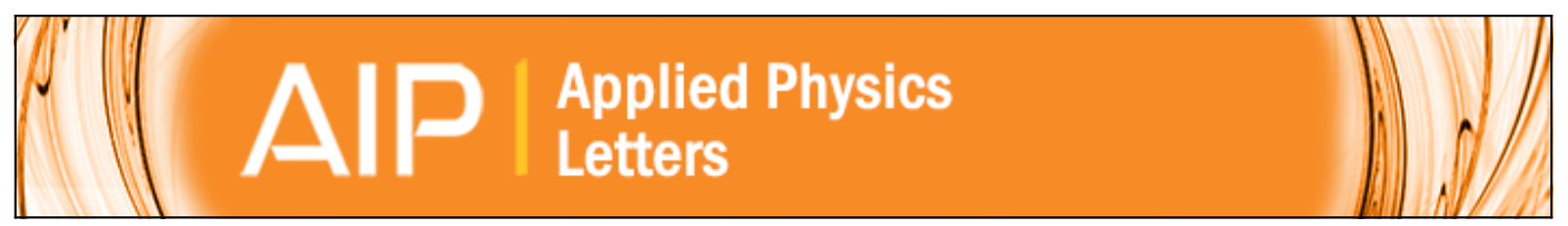

\title{
Reversible post-breakdown conduction in aluminum oxide-polymer capacitors
}

Qian Chen, Henrique L. Gomes, Paulo R. F. Rocha, Dago M. de Leeuw, and Stefan C. J. Meskers

Citation: Applied Physics Letters 102, 153509 (2013); doi: 10.1063/1.4802485

View online: http://dx.doi.org/10.1063/1.4802485

View Table of Contents: http://scitation.aip.org/content/aip/journal/apl/102/15?ver=pdfcov

Published by the AIP Publishing

\section{Articles you may be interested in}

Trapping of electrons in metal oxide-polymer memory diodes in the initial stage of electroforming

Appl. Phys. Lett. 97, 222106 (2010); 10.1063/1.3520517

Submicron gap capacitor for measurement of breakdown voltage in air

Rev. Sci. Instrum. 77, 034702 (2006); 10.1063/1.2185149

Electrical conduction transition and largely reduced leakage current in aluminum-doped barium strontium titanate thin films heteroepitaxially grown on $\mathrm{Ir} / \mathrm{MgO} / \mathrm{Si}(100)$

Appl. Phys. Lett. 86, 132902 (2005); 10.1063/1.1896448

Electric-field-induced conductance transition in 8-hydroxyquinoline aluminum ( Alq 3 )

J. Appl. Phys. 96, 3583 (2004); 10.1063/1.1778211

Contribution of interface capacitance to the electric-field breakdown in thin-film Al-AlO x - Al capacitors

Appl. Phys. Lett. 83, 2417 (2003); 10.1063/1.1613802

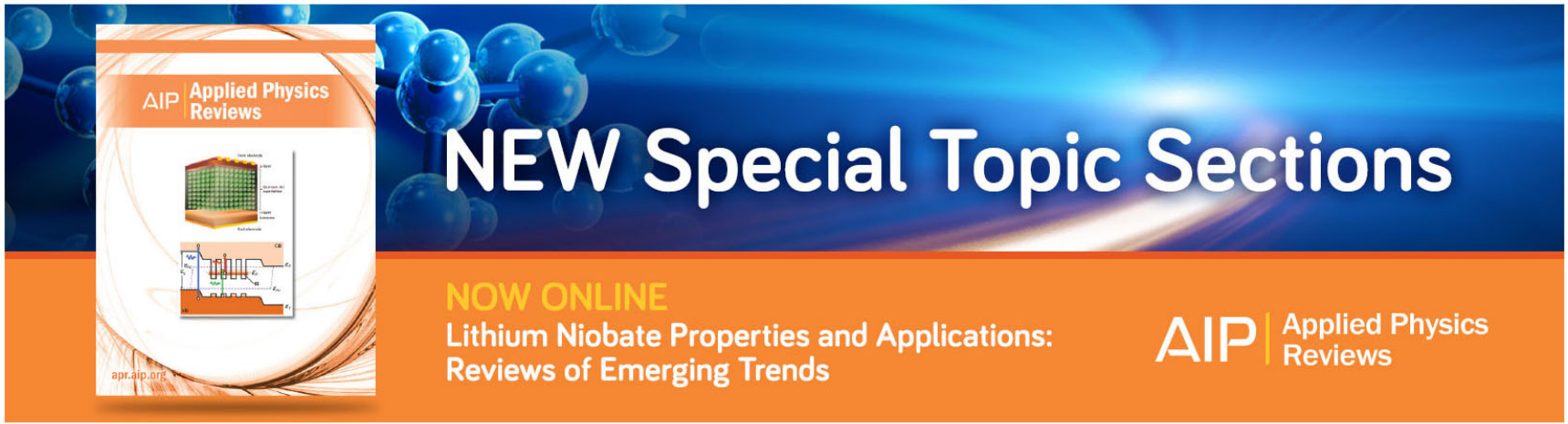




\title{
Reversible post-breakdown conduction in aluminum oxide-polymer capacitors
}

\author{
Qian Chen, ${ }^{1,2}$ Henrique L. Gomes, ${ }^{1,2, a)}$ Paulo R. F. Rocha, ${ }^{1,2}$ Dago M. de Leeuw, ${ }^{3}$ \\ and Stefan C. J. Meskers ${ }^{4}$ \\ ${ }^{1}$ Instituto de Telecomunicações, Av. Rovisco Pais 1, 1049-001 Lisboa, Portugal \\ ${ }^{2}$ Universidade do Algarve, Campus de Gambelas, 8005-139 Faro, Portugal \\ ${ }^{3}$ Max Planck Institut for Polymer Research, Ackermannweg 10, D-55128 Mainz, Germany \\ ${ }^{4}$ Molecular Materials and Nanosystems, Eindhoven University of Technology, P.O. Box 513, \\ 5600 MB Eindhoven, The Netherlands
}

(Received 20 January 2013; accepted 5 April 2013; published online 18 April 2013)

\begin{abstract}
Aluminum $/ \mathrm{Al}_{2} \mathrm{O}_{3} /$ polymer/metal capacitors submitted to a low-power constant current stress undergo dielectric breakdown. The post-breakdown conduction is metastable, and over time the capacitors recover their original insulating properties. The decay of the conduction with time follows a power law $(1 / t)^{\alpha}$. The magnitude of the exponent $\alpha$ can be raised by application of an electric field and lowered to practically zero by optical excitation of the polyspirofluorene polymer. The metastable conduction is attributed to formation of metastable pairs of oppositely charged defects across the oxide-polymer interface, and the self-healing is related to resistive switching. (C) 2013 AIP Publishing LLC [http://dx.doi.org/10.1063/1.4802485]
\end{abstract}

Dielectric breakdown (DB) of thin oxide layers has been investigated intensely because of the need to increase the reliability of metal-oxide-semiconductor field-effect transistors. There is a general consensus that DB occurs initially at one spot and results in localized currents. The Joule heating $^{1,2}$ in the DB spot leads to destruction of the oxide ${ }^{3}$ and eventually to device failure. ${ }^{4}$ Research has focused mainly on the pre-DB mechanism. ${ }^{5,6}$ The post-DB conduction mechanisms ${ }^{7,8}$ have received comparatively little attention. However, it has long been known that if excessive Joule heating is prevented, DB leads initially to bistable currentvoltage characteristics rather than directly to device failure. The bistability is interesting because of possible application in electronic data storage. In the research field of electronic data storage using oxides, controlled DB is often referred to as electroforming.

DB is usually assumed to be irreversible. ${ }^{9,10}$ This view is now under debate because recovery of DB was recently reported for $\mathrm{HfO}_{2}$ (Ref. 11) as well as for $\mathrm{SiO}_{2} \cdot{ }^{12,13}$ It has been proposed that restoration of the original insulating properties after oxide DB is due to the melting of the metallic filament by Joule heating ${ }^{14}$ or to annihilation of oxygen vacancies formed during DB. ${ }^{15} \mathrm{~A}$ similar phenomenon was recently reported for $\mathrm{Al} / \mathrm{Al}_{2} \mathrm{O}_{3}$ diodes and named "unforming,"16 but details were not presented.

Here we focus on the post-DB properties of aluminum oxide-polymer capacitors. We show that by subjecting the capacitor to low-power constant current stress (CCS) and by limiting the dissipation of electrical power, the DB is reversible. The decay of the post DB conduction to the original insulating conductance level follows a power-law decay kinetics $\sim(1 / t)^{\alpha}$. The exponent $\alpha$ is controlled by externally applied electric fields, and the value can be reduced by photoexcitation of the polyspirofluorene polymer. A model to

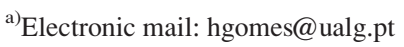

explain the self-healing is proposed. The consequences of switching on non-volatile memories are also discussed.

The capacitor structure (top of Fig. 1) consists of an $\mathrm{Al}$ bottom electrode, a sputtered layer of $\mathrm{Al}_{2} \mathrm{O}_{3}(20 \mathrm{~nm})$, a spirofluorene polymer $(80 \mathrm{~nm})$, and a $\mathrm{Ba} / \mathrm{Al}(5 \mathrm{~nm} / 100 \mathrm{~nm})$ top electrode that forms an ohmic contact with the polymer for electron injection. The devices with an active area of 1 and $9 \mathrm{~mm}^{2}$ were encapsulated to exclude $\mathrm{O}_{2}$ and $\mathrm{H}_{2} \mathrm{O}$. In all cases, positive bias voltage refers to the bottom $\mathrm{Al}$ electrode being positive with respect to the top Ba electrode. Current-voltage $(J-V)$ curves were obtained using a Keithley 487 picoammeter. CCS was maintained by a semiconductor parameter analyzer, Agilent 4156C. A blue Light Emitting Diode (LED) $\left(350 \leq \lambda \leq 650 \mathrm{~nm}, \lambda_{\max }=440 \mathrm{~nm}\right)$ was used as optical excitation source.

Fig. 1 shows DB of the capacitor under CCS. A current stress of $1 \mu \mathrm{A}$ is applied, and the voltage across the capacitor $\left(9 \mathrm{~mm}^{2}\right)$ is monitored as function of time. In the CCS method, the current is kept constant, and, therefore, changes in $d V / d t$ reflect changes in the diode capacitance $(C)$ with time, $C=I /(d V / d t)$. Initially, (first milliseconds) the voltage rises rapidly which shows that the diode has a low capacitance of $149 \mathrm{nF} / \mathrm{cm}^{2}$. This value is in agreement with the value of the series sum of the oxide capacitance $\left(C_{o x}=600 \mathrm{nF} / \mathrm{cm}^{2}\right)$ and polymer capacitance $\left(C_{\text {poly }}=200 \mathrm{nF} / \mathrm{cm}^{2}\right)$. As time progresses the $d V / d t$ slope saturates near the value of $600 \mathrm{nF} / \mathrm{cm}^{2}$, which corresponds to the estimated oxide capacitance.

The changes in capacitance before DB shows that the diode undergoes a change from a pure, capacitor-like behavior involving the oxide-polymer bilayer as dielectric, to behavior in which only the oxide layer acts as dielectric. The change in capacitance may be explained by the charge transport properties of the polymer. Electron transport is trap limited, and the effective resistance of the polymer layer depends on applied voltage. When a positive bias is applied, electrons are injected from the Ba electrode into the polymer and occupy trap sites. ${ }^{17}$ When the field is high enough, 


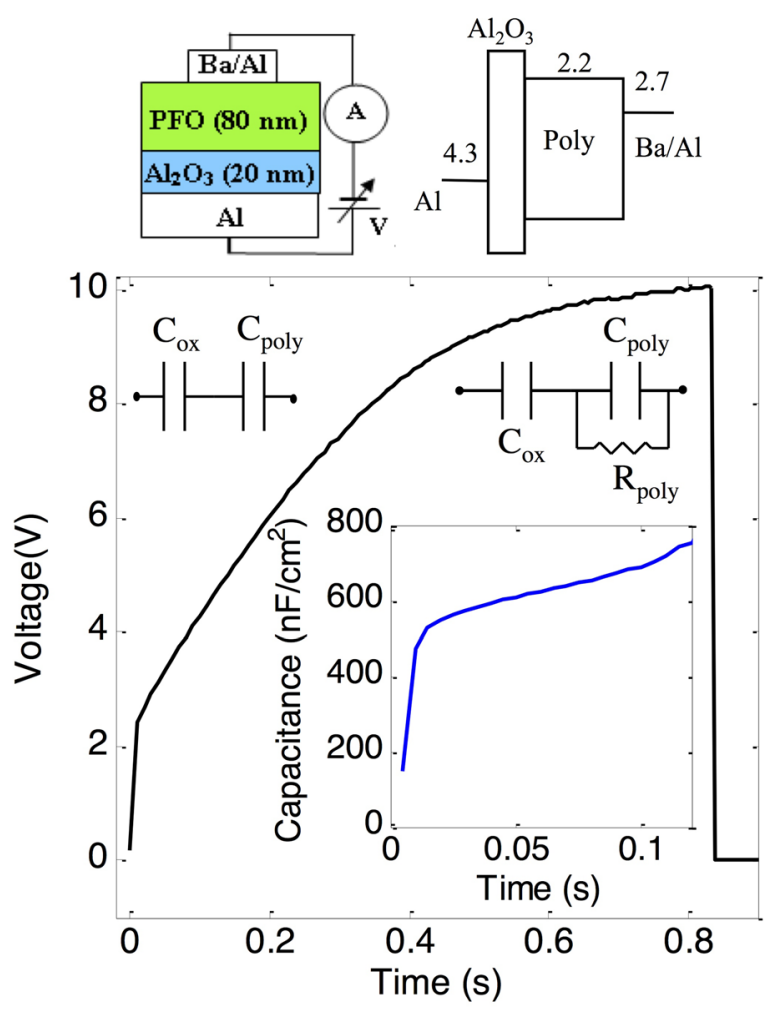

FIG. 1. Top: Layout of the capacitor and the schematic flat band diagram for the dielectric layers. Bottom: The voltage across the capacitor as function of time under CCS of $1 \mu \mathrm{A} / \mathrm{cm}^{2}$. An abrupt voltage drop is observed when voltage reaches $10 \mathrm{~V}$. Inset shows the corresponding change in capacitance estimated from the change in slope of the voltage.

electrons drift through the polymer and accumulate at the oxide/polymer interface. During the flow of this trapping current the diode behaves as a bilayer structure, and the change in $d V / d t$ reflects the $C_{o x}$ value. The inset of Fig. 1 shows the two equivalent circuits representing the limiting cases of the diode behavior on, respectively, a short timescale $(\mathrm{t}<0.05 \mathrm{~s}$ in Fig. 1) and intermediate timescale $(0.05<\mathrm{t}<0.8 \mathrm{~s})$. The dramatic change in behavior induced by dielectric breakdown (at $\mathrm{t}=0.8 \mathrm{~s}$ ) cannot be described by the simplified equivalent circuits shown.

During the application of constant current stress, the voltage across the capacitor rises with time until it reaches a critical value of around $10 \mathrm{~V}$ after about $0.8 \mathrm{~s}$. Upon reaching this critical voltage, a sudden drop in the voltage needed to maintain the constant current occurs, indicating a dielectric rupture. In the constant current method, increase in conduction is manifested by a decrease in voltage needed to sustain the programmed current and hence the DB results in enhanced conduction. The power consumption just before breakdown in Fig. 1 is estimated as $0.1 \mathrm{~mW} / \mathrm{cm}^{2}$. In comparison, the power used in electroforming the same type of capacitor into an electrically bistable resistive switching memory cell is much higher $\left(\sim 10 \mathrm{~mW} / \mathrm{cm}^{2}\right)$.

The increased conduction after breakdown is found to be a transient effect. Fig. 2(a) shows in a double logarithmic plot the time evolution of the electrical conduction of the capacitor probed by applying a constant bias of $0.5 \mathrm{~V}$. The conduction decreases over time following a power law dependence $I(t)=(1 / t)^{\alpha}$, where $\alpha$ is a constant. By inducing DB in the capacitor and holding the capacitor at a constant controlled bias
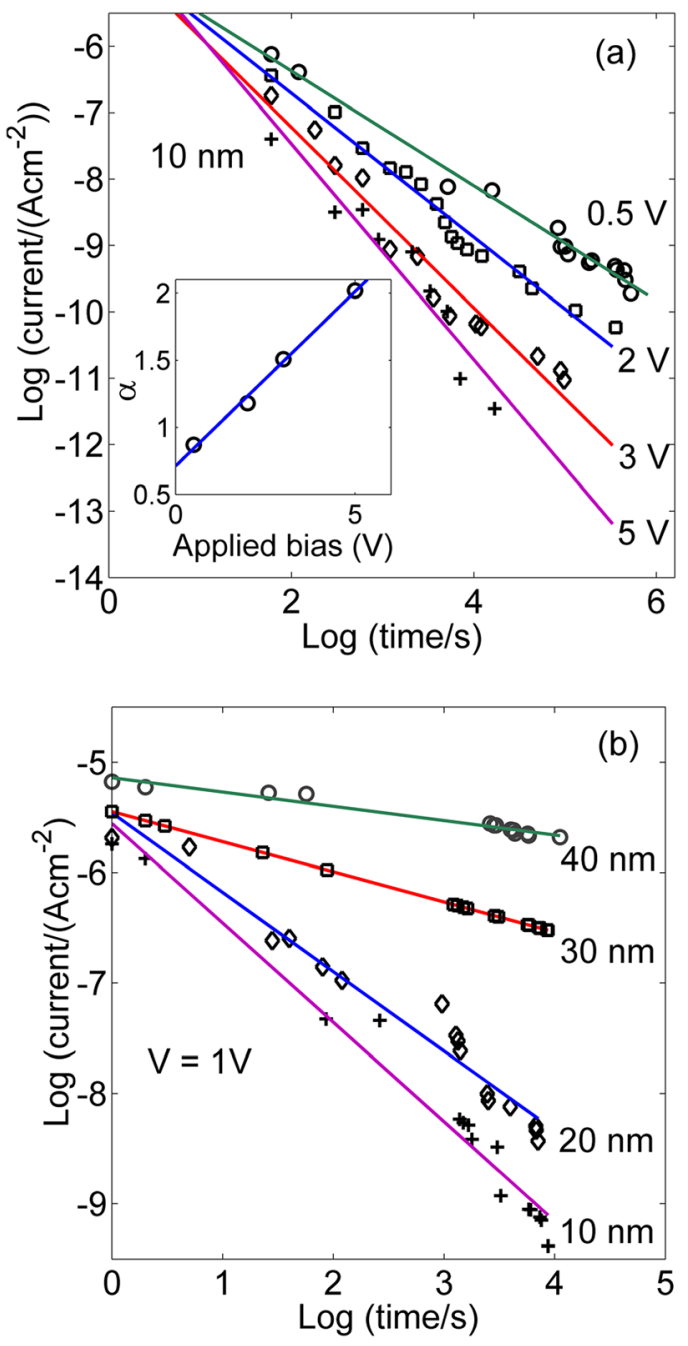

FIG. 2. (a) Current decay as function of time with different voltage biases $(0.5-5 \mathrm{~V})$ applied after the diode is electroformed by CCS. The current is measured at $0.5 \mathrm{~V}$. The inset shows that the power law $(\alpha)$ of current decay is directly proportional to the electric field. (b) Thickness dependence of current degradation monitored by voltage ramp (1 V) after forming by CCS, respectively.

voltage while intermittently probing the conduction at $0.5 \mathrm{~V}$ bias, the influence of an externally applied voltage bias on the decay of the conduction was investigated (Fig. 2(a)). The kinetics of the current decay should not be disturbed by the measurement procedure; for this reason, only one reading was done per data point, and the reading voltage $(0.5 \mathrm{~V})$ was applied for a short period of time $(\approx 1 \mathrm{~s})$. Therefore, error bars cannot be used. For all values of the bias voltage tested $(0.5,2$, 3 , and $5 \mathrm{~V}$ ), we find power law decay kinetics for the conduction, but, surprisingly, the magnitude of the exponent $\alpha$ increases with increasing bias. This indicates that the enhanced conduction in the capacitor after DB is due to a metastable arrangement of defects that is destabilized by application of forward bias voltage.

To confirm this hypothesis, capacitors with oxide thickness ranging from $10 \mathrm{~nm}$ to $40 \mathrm{~nm}$ were formed under CCS of $5 \mu \mathrm{A}$. After forming, the current at $1 \mathrm{~V}$ was then monitored as function of time. The corresponding decays are shown in Fig. 2(b). For thicker oxides the decay is slower. The reduced rate of decay of the conduction in capacitors with thicker oxide layer supports the hypothesis that the 
conduction arises from a metastable arrangement of defects that is destabilized by application of bias. This sensitivity towards applied fields indicates that the defects are charged. The recovery of the capacitor conductance to the pristine level is enhanced if an external voltage is applied or when there is an internal electric field across the oxide caused by trapped electrons at the oxide/polymer interface. In principle the oxide self-healing should be impeded if the trapped electrons are removed.

Previous studies ${ }^{18}$ have indicated that application of forward voltage to the capacitors results in trapping of electrons at the metal oxide/polymer interface. The trapped electrons can be removed by illuminating the polymer layer with photons having energy larger than the band-gap of the polymer $(3 \mathrm{eV})$. To determine whether trapped electrons play a role in the decay of the conduction after DB, we have studied the effect of irradiation with blue light on the decay of the conduction. First, DB was induced in the capacitor, followed by illumination for $1000 \mathrm{~s}$ directly after DB. The state of the capacitor was then monitored as function of time by measuring the current at $0.5 \mathrm{~V}$ up to $25 \mathrm{~h}$. We find that in the time period between DB and $25 \mathrm{~h}$ the current remains constant (Fig. 3). Then the capacitor was subjected to a voltage ramp up to $5 \mathrm{~V}$. This should restore the trapped electron density and trigger the self-healing mechanism. Indeed, after the application of $5 \mathrm{~V}$ the current monitored at $0.5 \mathrm{~V}$ decays with time following again the similar power law kinetics as shown in curve (a) of Fig. 3. After reaching the pristine state the same capacitor was electroformed again under identical conditions but not submitted to illumination. The current decay shown in Fig. 3(b) follows a power law kinetics with $\alpha=1$. The practically complete inhibition of the decay of the conduction after illumination indicates that electrons trapped at the oxide/polymer interface are involved in the decay process.

To rationalize the observed behavior, we propose the following mechanism for conduction induced by DB and the

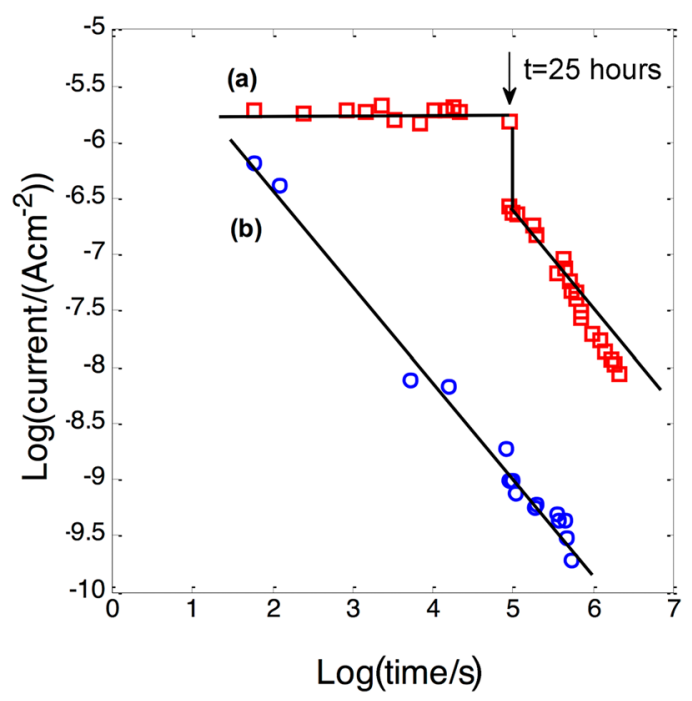

FIG. 3. After electroforming performed with CCS $(1 \mu \mathrm{A})$, (a) curve shows no current degradation with an illumination (1000s) of a blue LED applied shortly after the electroforming. The current decay (at $t=25 \mathrm{~h}$ ) restarts when a voltage ramp $(0-5 \mathrm{~V})$ is introduced. (b) The line shows the power law of current decay after electroforming, when the diode is kept in the dark and short-circuited. To minimize the effect of the applied bias the current decay was monitored at $0.5 \mathrm{~V}$. subsequent self-healing. Application of forward bias voltage to the capacitor before breakdown results in injection of electrons into the polymeric semiconductor. The electrons get trapped at the oxide/polymer interface. Consequently, the potential difference over the oxide layer becomes equal to the applied bias (Fig. 4(a)). In flat band condition when the applied bias voltage approaches the band-gap of the aluminum oxide, injection of positive charge carriers (holes) into the oxide becomes possible. ${ }^{19}$ We propose that the holes get trapped near the oxide/ polymer interface. The positive trapped holes are stabilized by the presence of the electrons on the other side of the interface, and the collection of holes and electrons forms an electrical bilayer. Because the capacitor is subjected to CCS, accumulation of holes in the oxide will continue until the electrical resistance is minimized. We note that the presence of an electrical twolayer structure results in a step in the potential profile along the capacitor. Once the conduction band edge offset between oxide and polymer is compensated by the potential step due to the bilayer and provided that the width of the bilayer is small enough to allow for electron tunneling, the electrical resistance will be minimized, and the hole injection process induced by the constant current stress should come to a halt (Fig. 4(b)). We note that for $\mathrm{ZnO}$-polymer diode structures, reduction of electrical resistance due to formation of an electrical bilayer at the oxide/polymer interface could be demonstrated..$^{20}$ Decay of the conduction induced by DB can be interpreted as recombination in the bilayer across the interface (Fig. 4(c)).

The recombination of positive charge carriers with number density $p$ may be assumed to be of Langevin-type, and the associated rate of recombination can be expressed as

$$
\frac{d p}{d t}=-\gamma n p
$$

where $n$ is the density of electrons and $\gamma$ is a rate constant. In order to find relations between the applied bias and carrier densities $n, p$, we treat the capacitor as an electrostatic system with three layers (Fig. 5(a)): oxide, double layer, and polymer with thicknesses $d_{\mathrm{ox}}, d_{\mathrm{dl}}$, and $d_{\mathrm{pol}}$ and dielectric
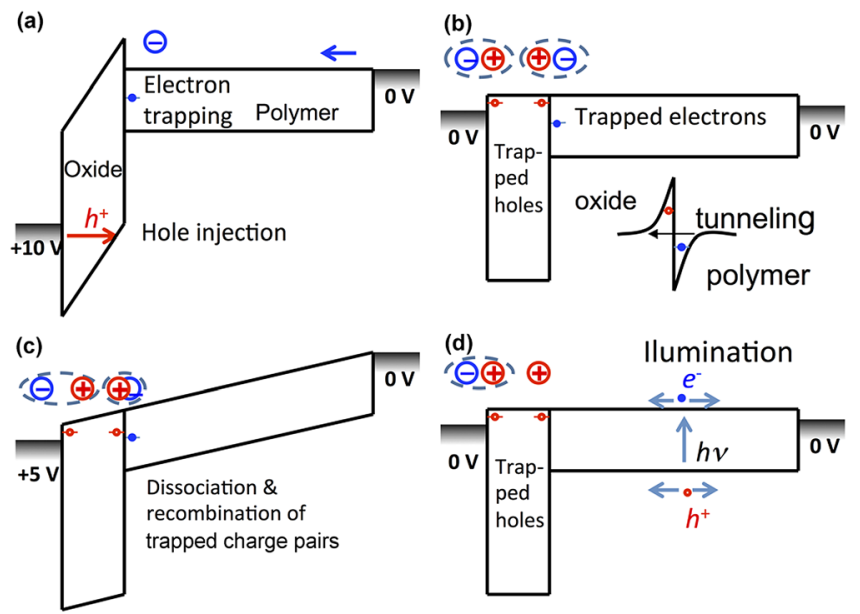

FIG. 4. (a) Injection and trapping of electrons at the oxide/polymer interface. (b) Injection and trapping of holes into the oxide. Trapped holes are stabilized by the presence of electrons (dipole layer). (c) Dissociation of the dipole layer caused by recombination. (d) Trapped electrons are removed by illumination the polymer layer with photons having energy larger than the band-gap of the polymer. 

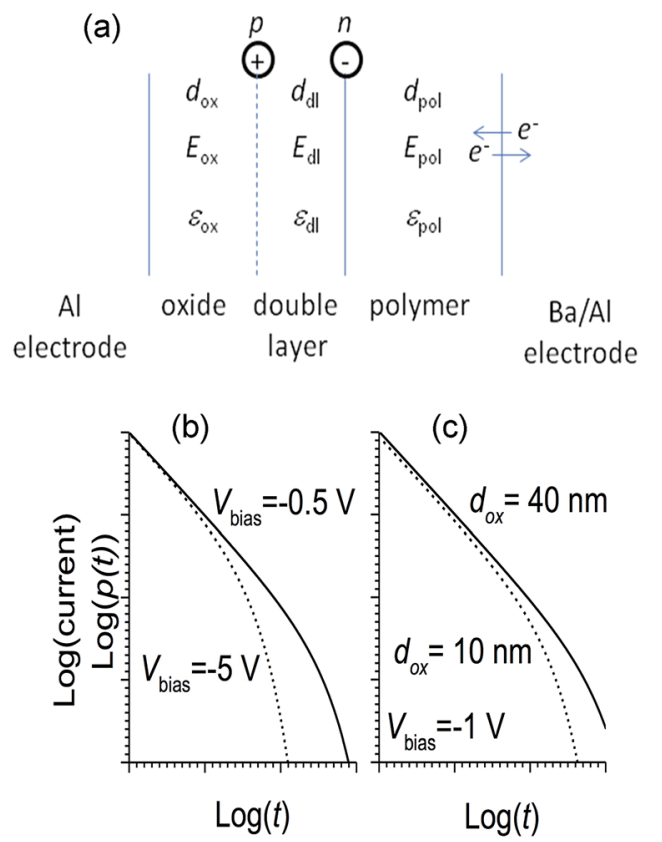

FIG. 5. (a) Schematic representation of the capacitor as a three layer electrostatic system with the oxide, the double layer, and the polymer layer. $d_{\mathrm{ox}}$, $d_{\mathrm{dl}}, d_{\mathrm{pol}}$ and $\varepsilon_{\mathrm{ox}}, \varepsilon_{\mathrm{dl}}, \varepsilon_{\mathrm{pol}}$ represent the thicknesses and the dielectric constants, respectively. Number of trapped holes in the oxide $p$ versus time as predicted by Eq. (7) for different bias voltages (b) and different oxide thickness (c). The current is expected to be proportional to the number of trapped holes.

constants $\varepsilon_{\mathrm{ox}}, \varepsilon_{\mathrm{dl}}$, and $\varepsilon_{\mathrm{pol}}$. At the oxide/double layer interface the positive carrier are located; at the interface between twolayer and polymer there are electrons.

From electrostatics we derive the following relations between applied bias $V_{\text {bias }}$ and carrier densities:

$$
\begin{gathered}
V_{b i a s}=V_{o x}+V_{d l}+V_{p o l}=V_{o x}+V_{d l}=d_{o x} E_{o x}+d_{d l} E_{d l}, \\
\varepsilon_{o x} E_{o x}-\varepsilon_{d l} E_{d l}=q_{e} p \\
-\varepsilon_{d l} E_{d l}=q_{e} n .
\end{gathered}
$$

From which we derive

$$
n=a p-a b V_{b i a s}
$$

where $\quad a=d_{\mathrm{ox}} \varepsilon_{\mathrm{dl}} /\left(d_{\mathrm{dl}} \varepsilon_{\mathrm{ox}}+d_{\mathrm{ox}} \varepsilon_{\mathrm{dl}}\right) \quad$ and $\quad b=\varepsilon_{\mathrm{ox}} /\left(q_{\mathrm{e}} d_{\mathrm{ox}}\right)$. Because typically $d_{\mathrm{dl}} \ll d_{\mathrm{ox}}$, one expects $a$ close to unity. Parameter $b$ is a capacitance per unit area per electron charge and is of the order $10^{16} \mathrm{~cm}^{-2} \mathrm{~V}^{-1} \cdot{ }^{18,19}$ Using these relations we can now get for the rate of decay

$$
\frac{d p}{d t}=-\gamma p\left(a p-a b V_{b i a s}\right)
$$

This relation can be solved by integrating in parts

$$
\int_{p\left(t_{0}\right)}^{p\left(t_{e}\right)} \frac{d p}{p\left(a p-a b V_{\text {bias }}\right)}=\int_{t_{0}}^{t_{e}}-\gamma d t=-\gamma\left(t_{e}-t_{0}\right) .
$$

The integral on the left hand side can be expressed in terms of the logarithmic function, and this gives an implicit expression for $p(t)$. Assuming that the conduction is proportional to the density of holes, we can then model current densities and the decay of the conductivity. Plots of the number of holes versus time according to Eq. (7) are given in Fig. 5. The model qualitatively reproduces the power law type behavior, and qualitatively reproduces the bias voltage and oxide thickness dependence of the decay.

In conclusion we have shown that in aluminum oxidepolymer capacitors constant current stress can induce dielectric breakdown. The amount of electric power that is dissipated is tightly controlled by the current limitation. Breakdown results in enhanced conductivity that decays overtime in a self-healing process. The dependence of the recovery of the insulating properties on applied bias and illumination indicates that the enhanced conduction is due to an electrical bilayer structure consisting of trapped holes in the oxide layer and electrons in the polymer layer and that the self-healing is due to recombination of charges comprising the bilayer structure.

We gratefully acknowledge Ton van den Biggelaar from Philips for preparing the devices. This work was financially supported by Dutch Polymer Institute (DPI) (Project No. 704) and by Fundação para Ciência e Tecnologia (FCT) through the Instituto de Telecomunicações (IT) and the project MemBrAiNN (PTDC/CTM-NAN/122868/2010).

${ }^{1}$ C. N. Berglund and N. Klein, Proc. IEEE 59, 1099 (1971).

${ }^{2}$ Y. Yagil, G. Deutscher, and D. J. Bergman, Physica A 207, 323 (1994).

${ }^{3}$ M. Bairanzade, Semiconductor Components Industries, LLC, 2003, Rev. 2.

${ }^{4}$ S. Lombardo, A. La Magna, C. Spinella, C. Gerardi, and F. Crupi, J. Appl. Phys. 86, 6382 (1999).

${ }^{5}$ T. W. Hickmott, J. Appl. Phys. 100, 083712 (2006).

${ }^{6} \mathrm{R}$. Waser, Microelectron. Eng. 86, 1925 (2009).

${ }^{7}$ E. Miranda, J. Appl. Phys. 96, 6940 (2004).

${ }^{8}$ X. Wu, K. L. Pey, N. Raghavan, W. H. Liu, X. Li, P. Bai, G. Zhang, and M. Bosman, Nanotechnology 22, 455702 (2011).

${ }^{9}$ J. W. McPherson, R. B. Khamankar, and A. Shanware, J. Appl. Phys. 88, 5351 (2000).

${ }^{10}$ S. Lombardo, J. H. Stathis, B. P. Linder, K. L. Pey, F. Palumbo, and C. H. Tung, J. Appl. Phys. 98, 121301 (2005).

${ }^{11}$ X. Wu, D. B. Migas, X. Li, M. Bosman, N. Raghavan, V. E. Borisenko, and K. L. Pey, Appl. Phys. Lett. 96, 172901 (2010).

${ }^{12}$ A. Crespo-Yepes, J. Martin-Martinez, R. Rodriguez, M. Nafria, and X. Aymerich, Microelectron. Reliab. 49, 1024 (2009).

${ }^{13}$ A. Crespo-Yepes, J. Martin-Martinez, A. Rothschild, R. Rodriguez, M. Nafria, and X. Aymerich, IEEE Electron Device Lett. 31, 543 (2010).

${ }^{14}$ X. Wu, K. L. Pey, G. Zhang, P. Bai, X. Li, W. H. Liu, and N. Raghavan, Appl. Phys. Lett. 96, 202903 (2010)

${ }^{15}$ H. D. Lee, B. Magyari-Köpe, and Y. Nishi, Phys. Rev. B 81, 193202 (2010).

${ }^{16}$ T. W. Hickmott, J. Appl. Phys. 111, 063708 (2012).

${ }^{17}$ T. W. Hickmott, J. Appl. Phys. 106, 103719 (2009).

${ }^{18}$ Q. Chen, B. F. Bory, A. Kiazadeh, P. R. F. Rocha, H. L. Gomes, F. Verbakel, D. M. de Leeuw, and S. C. J. Meskers, Appl. Phys. Lett. 99, 083305 (2011).

${ }^{19}$ B. F. Bory, H. L. Gomes, R. A. J. Janssen, D. M. de Leeuw, and S. C. J. Meskers, J. Phys. Chem. C 116, 12443 (2012).

${ }^{20}$ G. Lakhwani, R. F. H. Roijmans, A. J. Kronemeijer, J. Gilot, R. A. J. Janssen, and S. C. J. Meskers, J. Phys. Chem. C 114, 14804 (2010). 